\title{
Immunity and the emergence of individuality
}

\author{
Thomas Pradeu \\ Paris-Sorbonne University, IHPST \& Institut Universitaire de France \\ in Bouchard \& Huneman (eds.) From Groups to Individuals, MIT Press
}

\section{Draft September 2011}

\begin{abstract}
Since (Buss 1987), it has become clear that individuality is not to be considered as a given, but rather as something which needs to be explained. How has individuality emerged through evolution, and how has it subsequently been maintained? In particular, why is it that multicellular organisms appeared and persisted, despite the obvious interest of each cell of favoring its own replication? Several biologists see the immune system as one of the key components for explaining the maintenance of multicellular organisms' individuality. Indeed, the immune system exerts a constant surveillance on all the constituents of the organism, including "cheaters" like tumor cells, which favor their own replication at the expense of the whole organism. In most cases, the immune system eliminates those cheaters. This is the "immune surveillance" hypothesis, first suggested by Burnet and Thomas (Burnet 1957; Thomas 1959; Burnet 1970). In this paper, I account for recent findings on immune surveillance in order to determine the precise role of the immune system in the emergence and maintenance of individuality. This investigation gives rise to a critical question for the domain of biological individuality: should immunity be considered as something unique to multicellular organisms? If it is indeed unique, should the multicellular organism be considered as an individual to a higher degree than any other living entity (genomes, cells, groups, etc.)? If it is not unique, what precisely are the equivalents of the immune system in other living individuals (again, genomes, cells, groups, etc.)?
\end{abstract}

\section{Keywords}

Immune system, individual, organism, superorganism, evolutionary transition, symbiosis, philosophy of immunology. 


\section{Introduction: immunity and individuality}

It is crucial for biologists and philosophers alike to define what an individual is in the living world (Huxley 1912; Hull 1978, 1980, 1992; Buss 1987; Michod 1999; Santelices 1999; J. Wilson 1999; R. Wilson 2007; Godfrey-Smith 2009; Clarke 2010). Biological individuality is certainly one of the hottest topics nowadays in philosophy of biology and theoretical biology, with both predominantly or exclusively evolutionary approaches (Godfrey-Smith 2009; Gardner and Grafen 2009; Queller and Strassmann 2009; Folse and Roughgarden 2010; Clarke forthcoming), and approaches mixing evolution with the study of physiological or "metabolic" processes (Dupré and O'Malley 2009; Dupré 2010; Pradeu 2010; Pradeu 2012). It seems likely that a convincing account of biological individuality will need to embrace domains as diverse as evolutionary biology, genetics, developmental biology, immunology, perhaps neurology, etc.

Many biologists have insisted on the role of the immune system in the definition of biological individuality (Loeb 1930; Loeb 1945; Medawar 1957; Burnet 1960; Burnet 1969; Hamburger 1976; Buss 1987; Gould and Lloyd 1999; Michod 1999; Müller 2003; Cremer and Sixt 2009). Two main arguments are used to sustain this claim. The first argument pertains primarily to physiology, and seems rather intuitive - though I will show that it needs much clarification: the immune system, because it rejects some entities and accepts others, plays an important role in establishing the boundaries of the organism (Medawar 1957; Burnet 1969; Hamburger 1976; Gould and Lloyd 1999; Müller 2003; Cremer and Sixt 2009). The second argument pertains rather to evolutionary biology: it says that the immune system is one of the main "policing" mechanisms in living individuals, that is, one of the main mechanisms by which a high-level individual (typically, a multicellular organism) prevents the emergence of variants having a different fitness at a lower-level (typically, at the level of individual cells) (Michod 1999; Frank 1995, 1996, 2007; Rolff 2007).

In this paper I show the decisive role of the immune system in the emergence and maintenance of biological individuality. I demonstrate that the two arguments above are valid, but need to be made much more precise and be based on better evidence than has been done so far. I suggest immunity is central to defining what an organism is, to account for the unity of the organism despite the heterogeneity of its constituents (many of which are genetically "foreign"), to clarify the distinction between an organism and a biological individual, and finally to shed light on some evolutionary transitions.

A conceptual issue of crucial importance arises immediately. When some biologists talk about the "individual", they mean a multicellular organism (important examples include Lewontin 1970 and Buss 1987: viii). In sharp contrast, in the subsequent literature on units of selection understood as interactors (in particular Hull 1980; for a review, see Lloyd 2007), and then on evolutionary hierarchies and major transitions (Maynard-Smith and Szathmary 1995; Michod 1999; Okasha 2006; Godfrey-Smith 2009), an "individual" is an evolutionary individual, that is, an entity upon which natural selection acts as a whole. Within this framework, an "individual" may therefore refer to different levels in a biological hierarchy that comprises biological entities as different as genes, gene networks, genomes, organelles, cells, organisms, groups, etc. I endorse this view, and consider that the category "biological individual" is more inclusive than the category "organism" (see preliminary definitions below). But then a problem arises: I said that one of my claims was that immunity is crucial to understanding biological individuality, but it is not clear whether immunity can play a role at all these different levels of biological individuality. Is there any role for the immune system in defining the individuality of, say, a cell or a group? My strategy in this paper will be to focus on the role of the immune system in the definition of the multicellular organism's individuality, and then to raise the issue of the possible extension of this question to other biological entities, including unicellular organisms, individual cells in multicellular 
organisms, and "social organisms" such as some social insects. In the course of my argument, I will make clear why I do not endorse the now widespread view that "organism" is not a scientifically pertinent category (Dawkins 1982; Dupré and O'Malley 2009; Bouchard 2010, Bouchard this volume; Haber, this volume; for a defense of the organism concept, see Pepper and Herron 2008)

A related issue is to determine which organisms have an immune system. I will show that every organism does - vertebrates, invertebrates, plants, and even unicellulars.

Much confusion in the literature arises from the fact that no definition of the notions of individual and organism are given. As preliminary definitions, let me say that an individual in general is an entity that can be designated through a demonstrative reference (this $\mathrm{F}$ ), is separable, countable, has acceptably clear-cut spatial boundaries, and exhibits transtemporal identity, that is, the capacity to remain the "same" while changing through time. A biological individual is a living thing that fulfils these requirements. An organism is a functionally integrated living thing, highly organized, and made of interdependent parts. This later definition is admittedly vague (I will try to make it more precise), but we can easily see that, according to the definitions just given, a gene or an organelle, for instance, might be defined as biological individuals without being organisms. Other definitions could perhaps be adopted, but these are, I think, general enough to accommodate different views currently held about biological individuality.

In this paper, my aim is to show the fruitfulness of including immunity in the definition of biological individuality. I do this mainly through a close demonstration of the two claims above, that is, first the role of the immune system in establishing biological boundaries, and second the "self-policing" activity of the immune system. My strategy will be as follows. Section 2 shows the role of the immune system in establishing the boundaries of a biological individual. Section 3 makes clear why taking immunity into account sheds light on the individuation of every multicellular organism. In section 4 , I demonstrate that the recent revival of the "immune surveillance" hypothesis proves the validity of the claim (made, in particular, by Michod 1999) that the immune system is key in maintaining individuality at the level of the multicellular organism. Section 5 shows that the immune system was also certainly involved in the evolutionary emergence of individuality at the level of the multicellular organism. In section 6 I argue that every organism is heterogeneous, that is made of genetically different entities, and hence that the immune system controls not only genetically "self" constituents, but rather all the normal constituents of the organism, be they endogenous or exogenous (e.g., commensal and symbiotic bacteria). Finally, section 7 raises the issue of the possible extension of my conclusions from the case of the multicellular organism to other levels in the hierarchy of living individuals.

\section{The immune system and the establishment of the boundaries of the individual}

Many biologists and philosophers consider that the immune system plays a critical role in the definition of biological individuality because the immune system is pivotal to establishing the organism's boundaries. The key argument here is that the immune system offers a principle of inclusion (Pradeu 2010; Pradeu 2012), because it establishes what is rejected and what is not rejected by an organism. In so doing, the immune system determines which constituents stick together and thus are parts of one and the same organism. In addition to this exclusion-inclusion mechanism, the immune system is truly "systemic" in the sense that, contrary to many biological "systems" (respiratory, digestive, etc.) it exerts its activity everywhere in the organism, insuring the unity and the cohesiveness of the organism as a whole.

The general idea is put very clearly by Gould and Lloyd (1999), who say that the immune system plays a decisive role in the establishment of the spatial boundaries of 
organisms: "organisms are coherently bounded in space and kept recognizable in form by a physical skin that separates the self from the outside world, a distinction often buttressed by various devices - an immune system as the most prominent example - that can recognize and disarm or eliminate transgressors into the interior space". The same idea is expressed by Müller (2003), who, working on sponges, considers that an immune system is a prerequisite for biological individuality.

As the quotation from Gould and Lloyd (1999) suggests, the immunological selfnonself theory is certainly the best known framework used to explain how the immune system establishes the organism's boundaries. According to this theory (Burnet 1962; Burnet 1969), every foreign ("nonself") entity triggers an immune response, while no constituent of the organism ("self" constituent) triggers an immune response (except, of course, in pathological cases). Thus, the immune system is said to maintain the integrity of the organism via the recognition and rejection of every exogenous entity (as analyzed and criticized in Tauber 1994).

The self-nonself framework has been criticized from different perspectives, and several competing theories have been put forward (Pradeu 2012). Nevertheless, the common point between these different and competing approaches is that they all consider that the immune system is key to defining the boundaries of the organism.

Two problems arise with this account of biological individuality based on the action of the immune system. First, about the target of this account: it seems that the immune system says something about the organism, but not about every biological individual in general (a gene or a gene network, for instance, do not seem to have an immune system). In addition, it is often said that only higher vertebrates have an immune system; if this is true, then doesn't it make the immune system irrelevant for defining the individuality of the great majority of organisms living on Earth? I address this problem in section 3. Second, the immune system appears to shed light on one aspect of biological individuality, namely physiological unity and cohesiveness, but it is not yet clear whether the immune system can contribute to a better understanding of evolutionary individuality. Surely, physiological individuality and evolutionary individuality are related, but how exactly? In particular, is it possible to attribute a precise role to the immune system in evolutionary transitions, that is, in the emergence of a new evolutionary unit? These questions are addressed in sections 4 and 5.

\section{Immunity and organisms}

The preliminary definitions above have shown that the concept of a biological individual is larger than that of an organism. Therefore, one possible objection to the account given here is that it is too restrictive, as it addresses only the question of organismic individuality. For the moment, I accept this objection. I will show the fruitfulness of an immunological analysis of the organism's individuality, before asking what the contribution of immunology to a more general analysis of biological individuality may be (section 7 below).

Let us now deal with another, even more pressing, objection: isn't it well-known that only a small, even negligible, proportion of organisms have an immune system? Admittedly, higher vertebrates possess an immune system, but certainly invertebrates and plants do not? Here I show that this view - long held among immunologists - is now known to be utterly wrong. One of the main revolutions of current immunology is the redefinition of its scope (Pradeu 2009). Because of an almost exclusive focus on lymphocytes, immunologists have long considered that only Gnathostomata (i.e. jawed vertebrates) had a true immune system. But in fact, in all multicellular organisms in which investigations have been made, an immune system has been found, in the sense of a system of biochemically specific interactions leading to the rejection of some living entities. Here, by "organisms" I mean phenomenal organisms, 
that is, organisms as we perceive them and conceive them (later in this paper, I suggest a scientific definition of the organism, distinct from the phenomenal one). The fact that all multicellular organisms have an immune system should not be surprising, as they must all cope with pathogens - e.g. bacteria, viruses, helminthes, fungi. Well-studied examples include insects, in particular the Drosophila (Lemaitre and Hoffmann 2007), and plants (DeYoung and Innes 2006). A majority of organisms have what is called "innate" immunity (Janeway and Medzhitov 2002), as opposed to "adaptive" immunity, where a second encounter with one pathogen gives rise to a stronger and more rapid immune response. Nonetheless, the boundary between innate and adaptive immunity is difficult to establish (Vivier and Malissen 2005; Lanier and Sun 2009), and several forms of quicker and stronger response in the case of a second challenge with a pathogen have been documented (e.g. Kurtz and Franz 2003). In fact, even unicellulars have an immune system, a topic to which I shall return at the end of this text. Suffice to say for the moment that immunology does indeed deal with organisms, but its domain is extremely extensive nonetheless, as it includes all multicellular organisms, and possibly all organisms.

With this important precision in mind, we can now go back to the question raised above: the immune system seems to be useful in order to define the physiological individuality of present day organisms, but can it contribute in addition to a precise definition of evolutionary individuality? This question is crucial because the debate over biological individuality in the last decades has been framed mainly in evolutionary terms. In what follows, I show that immunity plays a decisive role in the definition of evolutionary individuality. Several biologists, especially Steven Frank (1996, 2002, 2007) and Richard Michod (1999) have suggested that the immune system is important in understanding the evolutionary transition from cells to multicellular organisms. Here I try to follow in their footsteps by analyzing the exact role of the immune system in this transition, and then to extend their framework, by asking whether the immune system helps us to understand other evolutionary transitions.

\section{The immune system and the maintenance of evolutionary individuality}

An evolutionary individual is an entity upon which natural selection acts as a whole (Hull 1980; 1992). On the basis of this definition, many biologists and philosophers have defended a hierarchical conception of evolutionary individuality, in which genes, gene networks, genomes, organelles, cells, organisms, groups, etc. may all, under proper circumstances, be considered as biological individuals (Hull 1980, 1992; Gould and Lloyd 1999; Gould 2002). A more stringent criterion, stemming from the literature on evolutionary transitions, says that evolutionary individuals must form lineages characterized by heritability of fitness (Michod 1999; Godfrey-Smith 2009).

A founding work was the book by Leo Buss, The Evolution of Individuality (Buss 1987). Buss shows that individuality is not a given, but something that has emerged in the course of evolution and was subsequently maintained. The crucial problem is the following: how, in the course of evolution, are higher level individuals constituted through the grouping of lower level individuals, and how is this new evolutionary individual subsequently maintained? In Michod's terms, why "lower-level units relinquish their claim to fitness, as it were, so that fitness may emerge at the new higher level" (Michod 1999: 6)? Michod says that the multicellular organism's harmony and integrity could be threatened by selection among cells, below the level of the organism (Michod 1999: 64). Therefore, some mechanisms probably make this threat unlikely, or less likely.

Following Buss and Michod's reasoning, two individualizing mechanisms can be suggested in order to explain the emergence and maintenance of a new, higher level of evolutionary individuality. The first mechanism consists in the prevention of the emergence 
of fitness conflicts at a lower level (it is an "ex ante" mechanism), by increasing the genetic homogeneity of the individual. In the case of the multicellular organism, the idea is that genetically homogenous cells will cooperate better than genetically heterogeneous ones (Buss 1987; Maynard-Smith and Szathmary 1995; Michod 1999: 108). The separation between germ cells and somatic cells is generally considered to be a mechanism of this type: somatic cells have no evolutionary interest in favoring their own fitness, as they are evolutionary dead ends (Godfrey-Smith 2009).

The second mechanism consists in the elimination of new variants favoring their own fitness (it is an "ex post" mechanism). This mechanism is often called "policing". The term "policing" may seem vague, and perhaps anthropomorphic, as this view is often formulated with expressions like "cooperation" (which is a larger concept than that of "policing"), "prevention against cheaters", etc. Yet the general argument can be straightforwardly put, by saying that a policing mechanism is one by which a biological entity eliminates lower level variants which favor their own fitness at the expense of the fitness of the whole organism (Frank 1995; Michod 1999).

The general idea behind these two individuating mechanisms (prevention of conflicts and policing of conflicts) is that the emergence of a new level of individuality always presupposes the partial repression of Darwinian processes (reproduction, heritability, differential fitness) at lower levels, what Peter Godfrey-Smith (2009) has vividly labeled "deDarwinization". I will now focus on policing mechanisms, and I will say a few words about the germ-soma separation (a prevention mechanism) below.

The clearest example of evolution of individuality is certainly that of the multicellular organism - the exclusive focus of Buss (1987) and the main focus of Michod (1999, 2005, 2007; Michod and Roze 2001). Why do cells in our bodies not replicate and increase their own fitness, even at the expense of the whole organism? Why do "cheaters" - that is, cells increasing their own fitness - not spread and disrupt the organism? According to Buss and Michod, several "policing" mechanisms eliminate new variants that may favor their own fitness. In the case of cancer cells, however, these mechanisms dysfunction, and we can observe that individual cells replicate and increase their fitness, indeed at the expense of the whole organism. Michod envisions two main policing mechanisms at the organism's level: the immune system and apoptosis (Michod 1999: 119). I will now analyze the role of the immune system, before going back to apoptosis.

In what sense can the immune system be described as a "policing" mechanism? The general idea is that the immune system constantly watches the constituents of the organism and exerts a control over intra-individual conflicts (Michod 1999: 119, 131-132). In particular, in many cases, the immune system detects tumors and eliminates them. I think Michod's hypothesis is very important and true, but unfortunately he gives few experimental arguments in support of it. Using recent literature in immunology, I would like now to explain why the research on "immune surveillance" shows that Michod is right when he says that the immune system is a policing mechanism.

The "immune surveillance" hypothesis was put forward by Burnet and by Thomas at the end of the 1950s (Burnet 1957; Thomas 1959). Burnet then gave a detailed account of this hypothesis in 1970 (Burnet 1970), where Burnet insists on the inspiring role of Thomas (Burnet 1970: 19). In the 1950s, it was undoubtedly a bold hypothesis, focusing on the evolution of the immune system. According to this hypothesis, the adaptive immune system has been selected through evolution for its capacity to constantly control the constituents of the organism and eliminate tumors. The hypothesis has three main aspects: i) tumors may arise constantly through the accumulation of genetic mutations, which lead to aberrant antigenic determinants at the surface of tumor cells; ii) the organism's lymphocytes can recognize these antigenic determinants and, in most cases, trigger the elimination of the 
tumor; iii) this capacity of the adaptive immune system to localize, recognize and destroy tumors is the main selective force which led to its emergence. Here is a telling illustration of Burnet's view in 1970: "There is no doubt whatever that once a cell line has emerged into malignancy, some type of general control has been abrogated, new anomalies in the genome arise freely including chromosomal changes and there is free scope for selection of variants of greater proliferative power" (Burnet 1970: 159); but, he adds, immunological surveillance usually acts before these changes, and normally prevents them from occurring (Burnet 1970: 160).

It may seem surprising that Burnet, the main artisan of the self-nonself theory (Burnet 1969), suggested a theory which depicts the immune system as rather "self-centered", that is, selected by evolution for its capacity to deal with "internal" entities, in this case tumors. Yet it should be kept in mind that Burnet considered tumors as "modified self", and therefore as a particular form of "foreign" antigens, that is, of "nonself" (Burnet 1970: 152).

The evidence in favor of the immune surveillance hypothesis was scarce. In the 1970s, several experiments, in particular those of Osias Stutman (Stutman 1974) tended to suggest that the immune surveillance hypothesis was wrong. In 1978, the hypothesis was considered dead (Dunn et al. 2002). But, after an eclipse of more than fifteen years, this hypothesis made a striking comeback, mainly through the demonstration of the role of interferon gamma (IFN$\gamma$ ) and of recombination activating genes (RAG) in preventing the development of tumors. As with many other subjects, Burnet (together with Thomas) had a remarkably adequate intuition, though the demonstration of the immune surveillance hypothesis took many years as, in fact, predicted by Burnet himself (Burnet 1970: 176-177).

Since the 2000s, the immune surveillance hypothesis has gained even stronger experimental support (Dunn et al. 2002; Pardoll 2003; Kupper and Fuhlbrigge 2004; Dunn, Koebel and Schreiber 2006; Koebel et al. 2007; Guerra et al. 2008; Cramer and Finn 2011; Fridman et al. 2011). Tumor cells trigger specific immune responses which, in a majority of cases, lead to their elimination. The components of the immune system involved in this elimination include, in particular, natural killer (NK) cells, T cells, interferon $\gamma$, macrophages, dendritic cells. Some have suggested the concept of "cancer immunoediting" as an extension of the concept of "immune surveillance": according to this concept, the immune system both eliminates the majority of tumors, and selects tumor variants that are better suited to survive in an immunologically intact environment - as it does when it selects bacterial or viral variants (Dunn et al. 2002).

Thus, immune surveillance exists, and is a critical activity of the immune system. It is not clear whether the elimination of tumors is the main evolutionary force that has shaped the immune system, as stated by Burnet and Thomas, but one can say at least that elimination of tumors is an important aspect of what an immune system does. In addition, and contrary to what has long been said, it seems unlikely that only vertebrates can develop cancers, as shown by recent work on Drosophila (Janic 2010) and other invertebrates (Robert 2010). More experimental data is needed on this subject, but it seems reasonable to suppose both that, in every multicellular organism, tumors may arise, and there exist some "policing" mechanisms which prevent the development of such tumors. My conclusion on this question is that immune surveillance as it is understood nowadays is exactly what was needed to anchor Michod's intuitions and models in convincing experimental data: these data show that the immune system plays a decisive role in the maintaining of individuality, preventing the emergence of conflicts of fitness at a lower level.

As we saw, Michod also envisions apoptosis as a policing mechanism. Apoptosis is physiological cell death (sometimes called "programmed cell death", or "cell suicide"), induced in defective, damaged or dangerous cells in the body (Kerr, Wyllie, Currie 1972; Wyllie, Kerr, Currie 1980; Vaux and Strasser 1996). Apoptosis helps to eliminate abnormal 
cells, and in particular tumor cells (Lowe, Cepero, Evan 2004). For different reasons, apoptosis can be seen not as another policing mechanism, but as one aspect of immune policing. Indeed, the key process of the elimination of apoptotic cells is accomplished by phagocytic cells, in particular macrophages, which are major components of the immune system (Jeannin, Jaillon and Delneste 2008; Elliott et al 2009). In addition, in several cases apoptosis is induced by immune cells, which have been activated by their interaction with the abnormal cells. This is true in particular of natural killer (NK) cells (Screpanti et al 2005; Vivier et al 2009). In any case, within the immune surveillance framework, it seems legitimate to include apoptosis in the general mechanism of policing of the body's cells by the immune system. And naturally, if adopted, this view further strengthens the claim that the immune system is key in maintaining individuality.

In addition, Michod sees policing mechanisms as only one set of mechanisms enabling a transition in individuality, along with germ-soma separation. Buss (1987) also insists on this aspect. He suggests that the sequestration of the germ line occurs only in a small fraction of the living world, but that it is precisely this fraction in which true biological individuals can be found.

My own view is that the role of germ-soma separation in biological individuality is much exaggerated. In a majority of organisms, in particular plants and colonial animals, this separation does not hold (Buss 1987; Clarke 2010, forthcoming). In contrast, the double immunological criterion (the establishment of boundaries thanks to the activity of the immune system, and the "policing" activity) does apply to plants and colonial organisms (Pradeu 2010). So immunity is a much stronger and more general criterion than other criteria, such as the germ-soma separation or the passage through a bottleneck, which are in fact rather rare (Clarke forthcoming). Because immunity plays this double role, and because it is widespread in so many different organisms, I suggest immunity is the most important mechanism to explain the evolution of the multicellular organism's individuality (as Peter Godfrey-Smith suggested to me, this view is close to that held by Müller 2003, though the arguments used to reach this conclusion are different)

In conclusion, the immune system is very important for maintaining evolutionary individuality in organisms, and this is true across phyla.

\section{The immune system and the emergence of evolutionary individuality}

Even though the immune system plays an important role in the maintenance of biological individuality, it is not clear whether it is involved in the emergence of individuality. In other words, immunity might have evolved as a late mechanism, strengthening the individuality of the multicellular organism, but not involved in its appearance in the first place. On the contrary, I suggest here that the immune system was probably critically involved in the emergence of the multicellular organism as a new biological individual.

Slime molds offer a model of the transition from unicellular to multicellular life. The social amoeba Dictyostelium discoideum adopts a double life: most of the time, it is unicellular, but when food becomes scarce, as many as 100000 cells gather and constitute a multicellular entity called a slug (Leslie 2007). This slug can then move to a different location, where $D$. discoideum generally returns to a unicellular mode of life. This social amoeba has fascinated many researchers both in the past (see Kessin 2001 for a review) and recently (Strassmann, Zhu and Queller 2000; Kessin 2001; Gregor et al. 2010; see also Bonner 2009 for a recent review). In an outstanding paper published in Science in 2007, it was shown that the social $D$. discoideum possesses an immune system (Chen, Zhuchenko and Kuspa 2007). Even though social amoebae feed on bacteria, they can also be infected by bacteria, including by its main pathogen Legionella pneumophila. Chen and colleagues show that a subset of $D$. discoideum cells, called "sentinel" cells, are specialized immune cells, 
which eliminate toxins and destroy bacterial pathogens. The sentinel cells circulate within the slug, and stick to pathogens when they meet them. The interaction is made via TirA, a TIR (Toll/interleukin-1 receptor) domain protein (TIR are typically involved in immune recognition, among other functions, both in plants and animals; O'Neill and Bowie 2007). These sentinel cells function to a large extent like neutrophils and macrophages in vertebrates. Importantly, $\mathrm{S}$ cells appear to be present in five other species of Dictyostelia, probably constituting a general characteristic of the social amoeba.

The discovery of Chen and colleagues strongly suggests that the development of specialized immune cells is a necessary preliminary step for the constitution of a multicellular organism. This early specialization seems essential to the transition to multicellularity. From an evolutionary point of view, it suggests that cells specialized in immune functions were present at the earliest steps of the transition from unicellular to multicellular life (Leslie 2007). It is not as surprising as it may appear at first sight, for an aggregation of cells with no immune system would certainly be prone to immediate disruption, both because of pathogens and of the appearance within the aggregate of some cells replicating and favoring their own fitness at the expense of the whole. An immune system that patrols the body and eliminates abnormal constituents seems indispensable for the construction and maintenance of the organism's cohesiveness.

The inference from observations on present social amoebae to the evolutionary transition from unicellularity to multicellularity is not straight-forward. Yet gathering data on organisms which oscillate between a unicellular and a multicellular way of life is overall the most appropriate way to make progress in the understanding of this evolutionary transition (Kirk 2005; Michod 2005; Leslie 2007). More work is certainly needed on other model organisms to better understand this transition, including the well-studied volvocine green algae (Kirk 2005; Michod 2005; Prochnik et al. 2010). But my intuition is that research to come will confirm the view presented here: an immune system eliminating abnormal components is strictly necessary for a collection of cells to become a unified and cohesive multicellular individual, or "organism."

The experimental and theoretical arguments offered here add to the analysis of Michod. In particular, as we saw, recent work on immune surveillance shows that Michod is right, and even that his conclusions can be radicalized by saying that the immune system plays a decisive role in the emergence and maintenance of individuality at the organism's level. I would like now to say more about the characterization of this cohesive multicellular organism. One of its main features is that it is not a genetically homogenous entity, and correlatively the criterion for immune elimination of abnormal constituents is not genetic homogeneity.

\section{Policing a heterogeneous organism}

Within the literature on evolutionary transitions, genetic homogeneity is seen as a crucial feature of a biological individual by a great majority of authors (Buss 1987; MaynardSmith and Szathmáry 1995; Michod 1999; Strassmann and Queller 2007). The general idea is that genetically homogeneous constituents will cooperate better than heterogeneous constituents, because they share a "common fate" (Dawkins 1976; Buss 1987; MaynardSmith and Szathmáry 1995; Michod 1999; Godfrey-Smith 2009). In particular, MaynardSmith and Szathmáry (1995) hold that the close kinship among cells explain their collaboration and the emergence of a new biological individual.

In fact, though, genetic homogeneity is not found in a great majority of organisms, both in plants and animals (Buss 1987; for a detailed analysis of individuality in plants, see Clarke forthcoming). More generally, it is not a necessary condition for the definition of an evolutionary individual (Michod 1999; Strassmann and Queller 2004; Godfrey-Smith 2009; 
Queller and Strassmann 2009). Many have suggested that true biological individuals, or best individualized entities, are those in which genetic homogeneity is found (Buss 1987). Yet I think this view must be rejected, because every multicellular organism is heterogeneous, that is, made of genetically different entities (Pradeu 2010). In particular, every multicellular organism (plant, invertebrate, vertebrate) hosts hundreds of billions of commensal and symbiotic bacteria (for example, it is estimated that the gut of a mammal hosts $10^{12}$ microorganisms; Garrett, Gordon and Glimcher 2010). Due to the massive presence of symbionts, every multicellular organism is a chimera. Many symbiotic bacteria play useful, even indispensable, roles in the body, in particular as far as digestion, immunity and development are concerned (Pradeu 2010, 2011). They are not simply present in the organism, but key functional constituents of it (O'Hara and Shanahan 2006; Garrett, Gordon and Glimcher 2010; Pradeu 2010). In addition, they are not confined to a few isolated parts of the organism; instead they are massively present in all the interfaces of the organism, which basically means almost everywhere in the organism (McFall-Ngai 2002; McFall-Ngai, Henderson and Ruby 2005). Moreover, they are not "invisible" to the organism, and in particular to the immune system, but on the contrary are in continuous interactions with it (Garrett, Gordon and Glimcher 2010). Finally, symbiotic bacteria often play a decisive evolutionary role, because they make a difference to the host's fitness and can be transmitted to the next generation (Pradeu 2010). I suggest that genetic homogeneity is not at all a criterion for biological individuality.

So every cohesive, functional organism is heterogeneous. Hence, immune surveillance exists, but precisely the immune system exerts its surveillance on the heterogeneous constituents of the body. It interacts constantly with the organism's normal constituents, be they genetically "self" constituents or symbiotic bacteria. Symbiotic bacteria are part of what immune surveillance targets: many bacteria must be seen as true constituents of the organism, that must be controlled by the immune system, exactly as "endogenous" constituents (Frank 1996). Normal bacteria are tolerated, but bacterial variants that proliferate abnormally in the organism are destroyed by the immune system (Eberl 2010).

Thus, what triggers an immune response of rejection is not genetic foreignness, but the expression of strongly unusual molecular patterns. This is the core assertion of the "continuity theory" (Pradeu and Carosella 2006a, 2006b; Pradeu 2012), according to which what triggers an immune response is the appearance of molecular patterns that are strongly different from those with which the immune system has interacted up to now, be they endogenous (as in the case of tumor cells, which are genetically self cells but which do trigger immune responses) or exogenous (as in the case of pathogenic bacteria, parasites, viruses, etc.)

The continuity theory constitutes an extension of the immune surveillance hypothesis of Burnet and Thomas (Pradeu 2012). The immune system constantly watches the organism's components, and responds against unusual (aberrant) proliferation of its constituents, whatever the origin of these constituents may be (a close meaning is given to the phrase "immune surveillance" by Ricklin et al. 2010). Incidentally, because the surveillance is exerted towards both endogenous and exogenous entities, a better, less anthropomorphic term for "policing" (suggested by Michod 1999 and now widely used) could be "elimination of uncontrolled reproducing living constituents".

To sum up our conclusions so far, a multicellular organism is made of heterogeneous constituents, some of them present from conception or birth, while others appear in the organism throughout its life (by "endogenous" or "exogenous" modifications, as in the case of newly integrated commensals or symbionts), to which a unity, a cohesiveness, must be given. Thus, a multicellular organism is characterized by the continuous construction of a cohesiveness through the permanent control of present constituents by the immune system. Immunity is key to insure the unity and cohesiveness of the organism. In my view, traditional 
evolutionary individuation needs to be complemented by an immunity-based individuation, because of the crucial role of the immune system in insuring the unity of different biological constituents (Pradeu 2010, 2012). I suggest to call biological individuals that are cohesively organized through an immune system "organisms". In this view, "organisms" are indeed special biological individuals, because they are individualized to a higher degree than other biological individuals. It is not that organisms as we see them ("phenomenal" organisms) are necessarily special, but that there are special things in the living world that we decide to call "organisms" (those which are cohesively organized through an immune system).

I will now end this paper by a discussion of an important question that I have up to now left aside: can immunology be useful to better understand biological individuals other than multicellular organisms? In other words, is the analysis of immunity relevant only for multicellular organisms, or is it also relevant beyond that case?

\section{Immune individuality beyond the level of the organism}

In this paper, I have talked mainly, indeed almost exclusively, about multicellular organisms. But if an organism is a biological individual that possesses an immune system, then the category of "organism" may be broader than expected initially. I will take here two important examples: first unicellulars, and second "superorganisms".

Contrary to what has been thought for many years, prokaryotic unicellulars appear to have an immune system. Because bacteria and archaea are constantly exposed to the threat of viruses (bacteriophages), it is not surprising that they possess some mechanisms to interact with them and eliminate them, but until recently the nature of these mechanisms remained unknown. In 2006, it was hypothesized that CRISPR-Cas is a system of adaptive immunity that integrates short genomic segments of selfish elements (viruses or plasmids) into specific loci in prokaryotic genomes and then employs these inserts to abrogate the replication of the cognate agents via a RNAinterference-like mechanism (Makarova et al. 2006; see also Makarova, Aravind, Wolf, Koonin 2011). In simpler terms, the suggestion was that bacteria and archaea possess an immune system which is based on a mechanism close to RNA interference. Major empirical evidence in favor of this hypothesis was found in 2007 (Barrangou et al. 2007). In a recent paper, Horvath and Barrangou (2010) explain that CRISPR is in fact not mechanistically analogous to eukaryotic RNA-interference, but is definitely an interference-based adaptive immune system. It is an immune system because it makes possible the specific recognition of viral sequences, and the elimination of the viruses; in addition, it can be called "adaptive," because a second encounter with the same virus leads to a quicker and stronger response. In addition, this protection can be transmitted to offspring. According to Horvath and Barrangou (2010), the CRISPR-cas system is massively present in Archaea (90\%) and probably less present in bacteria (40\%). There is now a growing consensus on the idea that the CRISPR-cas system must be seen as an adaptive immune system of microorganisms (van der oost et al. 2009; Garneau et al. 2010; Horvath and Barrangou 2010; Makarova, Haft et al. 2011).

Thus, it appears that unicellulars are biological individuals whose cohesiveness presupposes the constant action of an immune system. According to the view defended in the previous sections, it means that they are true "organisms". If this is correct, it means that the reflection offered above about the emergence and maintenance of individuality in multicellular organisms through the activity of an immune system needs in fact to be raised at the level of the much more ancient transition from independent replicators to the first prokaryotic cell. Because this transition is not very well known, and because basically nothing is known of the possible role of the immune system in this transition, I will leave this discussion for now, pending more experimental evidence in the near future. I think, though, that it raises the fascinating hypothesis that immunity has been a key element in both the 
evolutionary transition to multicellularity and the very ancient evolutionary transition to the first cell - often conceived of as the first "true" biological individual (Michod 1999; GodfreySmith 2009). It also suggests that each cell in multicellular organisms like us may have its own immune system. RNA silencing has been convincingly described as the "genome's immune system" (Plasterk 2002). Within this perspective, one can conceive a hierarchy of immunological individuals, or "organisms": a multicellular living thing like us is an organism in so far as it possesses an immune system, and in addition it comprises billions of cells, which themselves are organisms in so far as they each possess their own immune system. It is an attractive hypothesis, though it probably needs to be complemented by an analysis of the way in which the whole organism regulates immune responses at the level of each cell.

A second important question concerns so-called "superorganisms". A superorganism is a collection of organisms that can itself be described as an organism or a quasi-organism, because of some particular features, generally functional integration, cohesiveness and/or division of labor (EO Wilson 1971; Wilson and Sober 1989; Strassmann and Queller 2007; Hölldobler and Wilson 2008; Gardner and Graffen 2009) The most significant example is social insects, like some bees, termites and ants. Some authors even suggest to simply call the collective entities characterized by high cooperation and low conflict "organisms", arguing that "organismality" is only a question of degree, and that these collective entities possess a very high degree of organismality (Queller and Strassmann 2009). Because I have suggested that having an immune system is crucial for the cohesiveness of a biological individual, and even is indispensable to being an "organism", the question that must be raised now is the following: do the highly integrated collective entities often described as "superorganisms" possess an immune system?

Insects, and in particular social insects, have immune systems. In a majority of cases, the immune response in social insects occurs at the level of the individual insect. Yet colonylevel immunity may exist as well in some species (Cremer, Armitage and Schmid-Hempel 2007; Cremer and Sixt 2009). Indeed, in some cases, it appears that being part of a colony makes an important difference in the capacity to mount an immune response. In the termite $Z$. angusticollis, Traniello et al. (2002) recorded a significantly higher survivorship among nymphs that developed immunity as members of a group in comparison to isolated nymphs. Some bees have guards that control the nest's entrance and attack or exclude infected nestmates. It seems to be a case of colony-level immunity, and it is in those cases that it appears legitimate to talk about a true "social immunity" (Cremer, Armitage and SchmidHempel 2007). More research is needed on these forms of immunity in the many different "social" organisms. But it seems likely that, in some cases, the immune system of the colony will make it strongly cohesive, in such a way that the colony will qualify as an "organism". Additionally, these cases could shed light on the transition to multicellularity, and on evolutionary transitions more generally.

Again, more work is needed in this fascinating area. My only aim in this section has been to suggest that immunity may be decisive to understand not only the transition to the multicellular organism, but also the transition to the first prokaryotic unicellulars, and that to some "superorganisms".

\section{Conclusion}

Immunity plays a crucial role in defining biological individuality at the level of the multicellular organism, both because it delineates its boundaries (physiological individuation) and, through its "surveillance" activity, it has been indispensable in the emergence and maintenance of its cohesiveness (evolutionary individuation). The immune system is not the only feature that individualizes biological entities, but it individualizes them to an extremely high degree, since it constantly interacts with present constituents and rejects every abnormal 
component. Because of this essential role in insuring the unity and cohesiveness of a living thing, I suggest to call immunologically individualized biological entities "organisms". In my view, the role of immunity in the construction of biological individuality through evolution has been underestimated. I hope that this paper brings interesting data and arguments to fill in this gap.

\section{Acknowledgements}

I would like to thank Ellen Clarke, Peter Godfrey-Smith and Richard E. Michod for discussions on biological individuality.

\section{References}

Barrangou $\mathrm{R}$ et al. (2007) CRISPR provides acquired resistance against viruses in prokaryotes. Science 315: 1709-1712.

Bonner JT (2009) The Social amoebae: the biology of cellular slime molds. Princeton: Princeton University Press.

Bouchard F (2010) Symbiosis, Lateral Function Transfer and the (many) saplings of life. Biology and Philosophy (4): 623-641.

Burnet FM (1957) Cancer - a biological approach. Brit Med J 1: 841-847.

Burnet FM (1960) Immunological recognition of self. Nobel Lectures in Physiology or Medicine 3: 689-701.

Burnet FM (1969) Cellular immunology: Self and notself. Cambridge: Cambridge University Press.

Burnet FM (1970) Immunological surveillance. Oxford: Pergamon.

Buss L (1987) The evolution of individuality. Princeton: Princeton University Press.

Chen G, Zhuchenko O, Kuspa A (2007) Immune-like phagocyte activity in the social amoeba. Science 317: 678-681.

Clarke E. (2010) The problem of biological individuality. Biological Theory 5(4): 312-325.

Clarke E. (forthcoming) Plant Individuality: A Solution to the Demographer's Dilemma.

Cramer DW, Finn OJ (2011) Epidemiologic perspective on immune-surveillance in cancer.

Current Opinion in Immunology 23: 265-271.

Cremer S, Armitage SAO, Schmid-Hempel P (2007) Social immunity. Current Biology 17: R693-R702.

Cremer S, Sixt M (2009) Analogies in the evolution of individual and social immunity. Phil. Trans. R. Soc. B 364, 129-142.

Dawkins R (1976) The Selfish gene. Oxford: Oxford University Press.

DeYoung BJ, Innes RW (2006) Plant NBS-LRR proteins in pathogen sensing and host defense. Nature Immunology 7: 1243-1249.

Dunn GP, Bruce AT, Ikeda H, Old LJ, Schreiber RD (2002) Cancer immunoediting: from immunosurveillance to tumor escape. Nature Immunology 3(11), 991-998.

Dunn GP, Koebel CM, Schreiber RD (2006) Interferons, immunity and cancer immunoediting. Nature Reviews Immunology 6: 836-848.

Dupré J (2010) The polygenomic organism. Sociological Review 58 (s1): 19-31.

Dupré J, O'Malley M (2009) Varieties of living things: life at the intersection of lineage and metabolism. Philosophy and Theory in Biology 1 (online).

Eberl G (2010) A new vision of immunity: Homeostasis of the superorganism. Mucosal Immunology 3(5): 450-460.

Elliott MR et al (2009) Nucleotides released by apoptotic cells act as a find-me signal to promote phagocytic clearance. Nature 461: 282-287.

Folse 3rd HJ, Roughgarden J (2010) What is an individual organism? A multilevel selection perspective. The Quarterly review of biology 85 (4): 447. 
Frank SA (1995) George Price's contributions to evolutionary genetics. Journal of Theoretical Biology 175: 373-388.

Frank SA (1996) Host control of symbiont transmission: the separation of symbionts into germ and soma. The American Naturalist 148(6): 1113-1124.

Frank SA (2002) Immunology and evolution of infectious disease. Princeton: Princeton University Press.

Frank SA (2007) Dynamics of cancer: incidence, inheritance and evolution. Princeton: Princeton University Press.

Fridman WH et al. (2011) Immunosurveillance in human non-viral cancers. Current Opinion in Immunology 23: 272-278.

Gardner A, Grafen A (2009) Capturing the superorganism: a formal theory of group adaptation. Journal of Evolutionary Biology 22 (4): 659-671.

Garneau SE et al. (2010) The CRISPR/Cas bacterial immune system cleaves bacteriophage and plasmid DNA. Nature 468: 67-72.

Garrett WS, Gordon JI, Glimcher LH (2010) Homeostasis and inflammation in the intestine. Cell 140(6): 859-870.

Godfrey-Smith P (2009) Darwinian Populations and Natural Selection. New York: Oxford University Press.

Gould SJ, Lloyd E (1999) Individuality and adaptation across levels of selection: How shall we name and generalize the unit of Darwinism? Proceedings of the National Academy of Sciences USA 96(21): 11904-11909.

Gregor T, Fujimoto K, Masaki N, Sawai S (2010) The onset of collective behavior in social amoebae. Science 328: 1021-1025.

Guerra et al 2008. NKG2D-Deficient Mice Are Defective in Tumor Surveillance in Models of Spontaneous Malignancy. Immunity 28: 571-580.

Hamburger J (1978 [1976]) Discovering the individual. New York: Norton \& Company.

Hölldobler B, Wilson EO (2008) The superorganism: the beauty, elegance, and strangeness of insect societies. New York, NY: W. W. Norton.

Horvath P, Barrangou R (2010) CRISPR/Cas, the immune system of bacteria and archaea. Science 327: 167-170.

Hull D (1978) A Matter of Individuality. Philosophy of Science 45: 335-360.

Hull D (1980) Individuality and selection. Annual Review of Ecology and Systematics 11: 311-332.

Hull D (1992) "Individual", in Keller E.F. \& Lloyd E. (eds.), Keywords in Evolutionary Biology. Cambridge, MA: Harvard University Press.

Janeway CA, Medzhitov R (2002) Innate immune recognition. Annual Review of Immunology 20: 197-216.

Janic A et al. (2010) Ectopic Expression of Germline Genes Drives Malignant Brain Tumor Growth in Drosophila, Science 330: 1824-1827.

Jeannin P, Jaillon S, Delneste Y (2008) Pattern recognition receptors in the immune response against dying cells. Current opinion in immunology 20: 530-537.

Kerr JFR, Wyllie AH, Currie AR (1972) Apoptosis: a basic biological phenomenon with wide-ranging implications in tissue kinetics. Br. J. Cancer 26: 239-57

Kessin RH (2001) Dictyostelium: evolution, cell biology, and the development of multicellularity. Cambridge: Cambridge University Press.

Kirk DL (2005) A twelve-step program for evolving multicellularity and a division of labor. BioEssays 27: 299-310.

Koebel CM et al. (2007) Adaptive immunity maintains occult cancer in an equilibrium state, Nature 450: 903-907. 
Kupper TS, Fuhlbrigge RC (2004) Immune surveillance in the skin: mechanisms and clinical consequences. Nature Reviews Immunology 4: 211-222.

Kurtz J, Franz K (2003) Evidence for memory in invertebrate immunity. Nature 425: 37-38. Lanier L, Sun J (2009) Do the terms innate and adaptive immunity create conceptual barriers? Nature Reviews Immunology 9: 302-303.

Lemaitre B, Hoffmann J (2007) The Host defense of Drosophila melanogaster. Annual Review of Immunology 25: 697-743.

Leslie M (2007) A slimy start for immunity? Science 317: 584.

Lewontin R (1970) The units of selection. Annual Review of Ecology and Systematics 1: 118.

Lloyd EA (2007) Units and levels of selection, in The Cambridge companion to the philosophy of biology, ed. D.L. Hull and M. Ruse, 44-65. Cambridge: Cambridge University Press.

Loeb L (1930) Transplantation and individuality. Physiological Review 10: 547-616.

Loeb L (1945) The biological basis of individuality. Springfield: Thomas.

Lowe SW, Cepero E, Evan G (2004) Intrinsic tumour suppression. Nature 432: 307-315.

McFall-Ngai M (2002) Unseen forces: The influence of bacteria on animal development. Developmental Biology 242: 1-14.

McFall-Ngai M, Henderson B, Ruby EG, eds. (2005) The Influence of Cooperative Bacteria on Animal Host Biology. Cambridge, UK: Cambridge University Press.

Makarova KS et al. (2006) A putative RNA-interference-based immune system in prokaryotes: Computational analysis of the predicted enzymatic machinery, functional analogies with eukaryotic RNAi, and hypothetical mechanisms of action. Biology Direct 1(7).

Makarova KS, Aravind L, Wolf YI, Koonin EV (2011) Unification of Cas protein families and a simple scenario for the origin and evolution of CRISPR-Cas systems. Biology Direct 6: 38.

Makarova KS, Haft DH, Barrangou R, Brouns SJJ, Charpentier E, Horvath P, Moineau S, Mojica FJM, Wolf YI, Yakunin AF, van der Oost J, Koonin EV (2011) Evolution and classification of the CRISPR-Cas systems. Nature Reviews Microbiology 9: 467-477.

Maynard-Smith J, Szathmáry E (1995) The Major Transitions in Evolution. New York: Oxford University Press.

Medawar PB (1957) The uniqueness of the individual. London: Methuen.

Michod RE (1999) Darwinian dynamics: evolutionary transitions in fitness and individuality, Princeton, Princeton University Press.

Michod RE (2005) On the transfer of fitness from the cell to the multicellular organism. Biology and Philosophy 20: 967-987.

Michod RE (2007) Evolution of individuality during the transition from unicellular to multicellular life. Proceedings of the National Academy of Sciences USA 104(1): 8613-8618.

Michod R, Roze D (2001) Cooperation and conflict in the evolution of multicellularity. Heredity 86: 1-7.

O'Hara AM, Shanahan F (2006) The gut flora as a forgotten organ. EMBO Reports 7(7): 688-693.

O'Neill LAJ, Bowie AG (2007) The family of five: TIR-domain-containing adaptors in Tolllike receptor signalling. Nature Reviews Immunology 7: 353-364.

Okasha S (2006) Evolution and the levels of selection. New York: Oxford University Press.

Pepper JW, Herron MD (2008) Does biology need an organism concept? Biol. Rev. 83: 621627.

Plasterk RH (2002) RNA silencing: the genome's immune system. Science 296: 1263-1265.

Pradeu T (2009) Immune system: 'Big Bang' in question. Science 325(5939): 393. 
Pradeu T (2010) What is an organism? An immunological answer. History and Philosophy of the Life Sciences 32: 247-268.

Pradeu T (2011) A mixed self: the role of symbiosis in development. Biological Theory 6(1). Pradeu (2012) The Limits of the self: Immunology and biological identity. New York: Oxford University Press.

Pradeu T, Carosella ED (2006a) The self model and the definition of biological identity in immunology. Biology and Philosophy 21: 235-252.

Pradeu T, Carosella ED (2006b) On the definition of a criterion of immunogenicity. Proceedings of the National Academy of Sciences USA 103(47): 17858-17861.

Prochnik SE et al (2010) Genomic Analysis of Organismal Complexity in the Multicellular Green Alga Volvox carteri. Science 329: 223-226.

Queller DC, Strassmann JE (2009) Beyond society: the evolution of organismality. Philosophical Transactions of the Royal Society B: Biological Sciences 364 (1533): 3143.

Ricklin D, Hajishengallis G, Yang K, Lambris JD. 2010. Complement: a key system for immune surveillance and homeostasis. Nature Immunology 11(9): 785-797.

Robert J (2010), Comparative study of tumorigenesis and tumor immunity in invertebrates and nonmammalian vertebrates. Developmental and Comparative Immunology 34: 915-925.

Rolff 2007. Why did the acquired immune system of vertebrates evolve? Developmental and Comparative Immunology 31 (2007) 476-482

Santelices B (1999) How many kinds of individual are there? Trends in Ecology \& Evolution 14 (4): 152-155.

Screpanti V, Wallin RPA, Grandien A, Ljunggren HG (2005) Impact of FASL-induced apoptosis in the elimination of tumor cells by NK cells. Molecular Immunology 42: 495-499.

Strassmann JE, Queller DC (2004) Genetic conflicts and intercellular heterogeneity. Journal of Evolutionary Biology 17: 1189-1191.

Strassmann JE, Queller DC (2007) Insect societies as divided organisms: the complexities of purpose and crosspurpose. Proceedings of the National Academy of Sciences USA 104, 86198626.

Strassmann JE, Zhu Y, Queller DC (2000) Altruism and social cheating in the social amoeba, Dictyostelium discoideum. Nature 408: 965-967.

Stutman O (1974) Tumor development after 3-methylcholanthrene in immunologically deficient athymic-nude mice. Science 183: 534-536.

Tauber AI (1994) The Immune self: theory or metaphor? Cambridge: Cambridge University Press.

Thomas L. (1959) Discussion in Cellular and Humoral Aspects of the Hypersensitive States, ed. H.S. Lawrence, New York: Hoeber-Harper, 529-532.

Traniello JFA, Rosengaus, RB, Savoie K (2002) The development of immunity in a social insect: evidence for the group facilitation of disease resistance. Proceedings of the National Academy of Sciences USA 99: 6838-6842.

van der Oost J. et al. (2009) CRISPR-based adaptive and heritable immunity in prokaryotes.

Trends in Biochemical Sciences 34(8): 401-407.

Vaux DL, Strasser A (1996) The molecular biology of apoptosis. Proc. Natl. Acad. Sci. USA 93: 2239-44

Vivier E, Malissen B (2005) Innate and adaptive immunity: Specificities and signaling hierarchies revisited. Nature Immunology 6(1): 17-21.

Vivier E et al (2009) Innate or Adaptive Immunity? The Example of Natural Killer Cells. Science 331: 44-49.

Wilson DS, Sober E (1989) Reviving the superorganism. Journal of Theoretical Biology 136: 337-356. 
Wilson EO (1971) The Insect Societies. Cambridge, MA: Belknap Press of Harvard University Press.

Wilson J (1999) Biological individuality: the identity and persistence of living entities. Cambridge: Cambridge University Press.

Wilson R (2007) The biological notion of individual, Stanford Encyclopedia of Philosophy (online).

Wyllie AH, Kerr JFR, Currie AR (1980) Cell death: the significance of apoptosis. Int. Rev. Cytol. 68:251-306. 